\title{
Research Article \\ Design of a CPW-Fed Band-Notched UWB Antenna Using a Feeder-Embedded Slotline Resonator
}

\author{
Amin M. Abbosh \\ School of Information Technology and Electrical Engineering, The University of Queensland, St. Lucia, Qld 4072, Australia
}

Correspondence should be addressed to Amin M. Abbosh, abbosh@itee.uq.edu.au

Received 1 August 2007; Revised 21 January 2008; Accepted 10 March 2008

Recommended by Hans Schantz

A complete design method for a compact uniplanar ultra-wideband antenna with subband rejection capability is presented. A slotline resonator is incorporated in the coplanar waveguide feeder of the antenna to act as a bandstop filter, hence enabling the rejection of any undesired band within the passband of the antenna. Two samples of the proposed antenna were designed and manufactured. One of the developed antennas does not contain a resonator, whereas the other contains a slotline resonator. The designed antennas feature a compact size of $27 \mathrm{~mm} \times 27 \mathrm{~mm}$. Results of the simulation and measurement show that the designed antennas have a bandwidth from $3 \mathrm{GHz}$ to more than $11 \mathrm{GHz}$. The results also reveal that the use of the resonator in the feeder of the antenna efficiently rejects any undesired subband, such as the 4.9-5.9 GHz band assigned for IEEE802.11a and HIPERLAN/2. The gain of the antennas with the resonator is about $2.2 \mathrm{dBi}$ at the passband, while it is less than $-8 \mathrm{dBi}$ at the rejected subband.

Copyright (C) 2008 Amin M. Abbosh. This is an open access article distributed under the Creative Commons Attribution License, which permits unrestricted use, distribution, and reproduction in any medium, provided the original work is properly cited.

\section{INTRODUCTION}

Ultra-wideband (UWB) is a short range communications technology. It has gained a lot of popularity among researchers and the wireless industry after the FCC permitted the marketing and operation of UWB within the range $3.1 \mathrm{GHz}$ to $10.6 \mathrm{GHz}$ [1]. The global interest in the UWB technology is increasing very fast due to the capability of this license exempt wide bandwidth system to yield low cost, low energy, short range, and extremely high capacity wireless communication links.

In addition to the requirement of the $3.1-10.6 \mathrm{GHz}$ bandwidth, the UWB antennas should also have the capability to reject any interference with existing wireless networking technologies such as the subband $4.9-5.9 \mathrm{GHz}$ for IEEE 802.11a in the USA, and HIPERLAN/2 in Europe. Therefore, UWB antennas with notched characteristics at certain bands are desired. The ability to provide this function in the antenna can significantly relax the requirements imposed upon the filtering electronics within the wireless communication system.

One of the first attempts to design a band-notched ultrawideband antenna was presented in [2]. The design of a
UWB antenna with a good impedance matching over the desired band was achieved using a genetic algorithm optimization code. A manual trial-and-error approach was then utilized to modify the design towards a band-notched antenna. In a later stage, the authors of [2] extend their optimization technique to improve the radiation pattern of their band-notched design [3].

A systematic method for designing frequency notched ultra-wideband antennas was presented in [4]. By deliberately introducing a narrow band resonant structure, an antenna may be made capable of rejecting particular frequencies. This technique is useful for creating UWB antennas with narrow frequency notches, or for creating multiband antennas.

Reviewing the literature shows that there are many different methods used to achieve the band-notched function. The conventional methods are cutting a slot of different shapes on the radiating patch [5-7], inserting a slit on the patch [8], embedding a quarter-wavelength tuning stub within a large slot on the patch [9], putting parasitic elements near the radiator as filters to reject the limited band [10], or introducing a parasitic open-circuit element, rather than modifying the structure of the antenna's tuning stub [11]. 
This paper describes a different design method for a UWB antenna with a subband rejection capability. Instead of modifying the radiator as with the proposed methods in the literature, a slotline resonator is incorporated in the coplanar waveguide feeder of the antenna to filter out any undesired subband with negligible effect on the passband of the antenna. The method presented in this paper is used to design a uniplanar UWB antenna which rejects the 4.9$5.9 \mathrm{GHz}$ band, which is assigned for the IEEE802.11a and HIPERLAN/2. The results of simulations and measurements for two manufactured antennas (one without a resonator and the other with a slotline resonator) are presented in order to prove the validity of the proposed method.

\section{DESIGN}

The configuration of the proposed UWB antenna with the capability of rejecting frequencies over a certain subband is illustrated in Figure 1. The radiating structure is formed by the connection of half an ellipse with a rectangular patch. The antenna is assumed to be fed using a coplanar waveguide (CPW) to enhance its broadband characteristics. The ground plane, which is located around the CPW feeder, is in the shape of half an ellipse.

The design procedure starts by finding dimension of the antenna feeder to give $50 \Omega$ characteristic impedance $\left(Z_{o}\right)$. This can be achieved using the following equations [12]:

$$
\begin{gathered}
Z_{o}=\frac{30 \pi}{\sqrt{\varepsilon_{e}}} \frac{K^{\prime}(k)}{K(k)}, \\
k=\frac{s}{s+2 w_{c}}, \\
\varepsilon_{e}=\frac{\varepsilon_{r}+1}{2}[A+B], \\
A=\tanh \left\{1.785 \log \left(h / w_{c}\right)+1.75\right\}, \\
B=\frac{k w_{c}}{h}\left\{0.04-0.7 k+0.01\left(1-0.1 \varepsilon_{r}\right)(0.25+k)\right\},
\end{gathered}
$$

where $K(k)$ is the first kind elliptical integral and $K^{\prime}(k)=$ $K\left(\sqrt{1-k^{2}}\right), s$ is the central conductor width, whereas $w_{c}$ is the slot width of the CPW, $h$ is the substrate thickness, and $\varepsilon_{r}$ is the dielectric constant of the substrate.

Depending on the lowest frequency of operation $\left(f_{l}=\right.$ $3.1 \mathrm{GHz})$, thickness of the substrate and its dielectric constant, width $(w)$, and length $(l)$ of the antenna structure are calculated as

$$
w=l=\frac{c}{2 f_{l} \sqrt{\varepsilon_{r}}},
$$

where $c$ is the speed of light. The antenna is assumed to be in the $x y$ plane, with the dimension $w$ extending along the $x$ axis. Note that the length and width of the antenna structure, according to (2), are equal to half of the wavelength inside the substrate medium.

It is worthwhile to mention that (2) gives an accurate estimation of the required dimension of the antenna for a

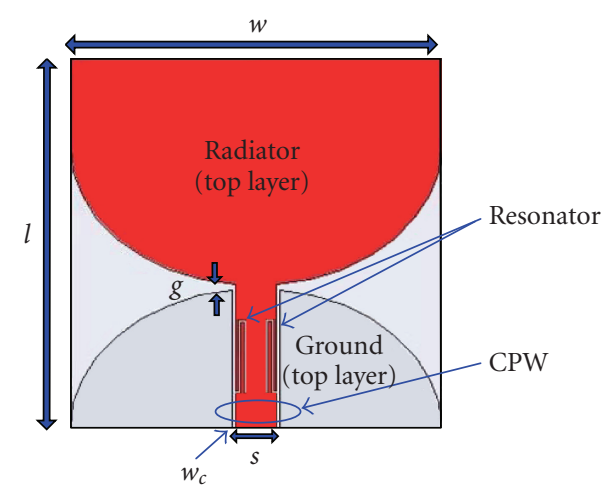

(a)

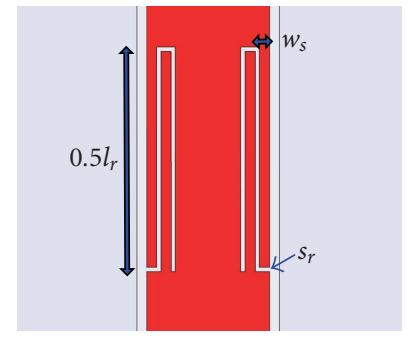

(b)

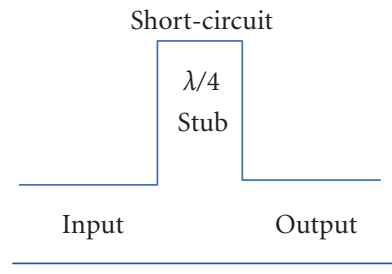

(c)
Figure 1: (a) Configuration of the proposed antenna, (b) details of the slotline resonator, and (c) the transmission-line model of the resonator.

low value of the dielectric constant of the substrate, that is, less than 4 . If it is required to find a rough estimation of the required dimension for any value of the dielectric constant, the following formula can be used:

$$
w=l=\frac{c}{2 f_{l} \sqrt{\left(\varepsilon_{r}+1\right) / 2}} .
$$

The ground plane of the antenna, which is located around the CPW, is half of an ellipse with major diameter equal to $(w)$. The secondary diameter of the ground plane can be chosen to be around $(0.5 w)$.

The radiating structure, which is located at a distance $g$ from the end of the ground plane, consists of two parts. The first part, which is connected directly with the feeder, is half an ellipse with the same dimensions as for the ground plane. The second part is a rectangular patch which extends from the end of the half ellipse till the end of the substrate. Parametric analysis on the best value for $g$ indicates that it should be less than thickness of the substrate $h$ in order to get the widest bandwidth. In the design procedure of this paper, $g$ is assumed to be equal to $(h / 3)$.

Note that the above choice for dimension of the radiator and the ground plane results in a horizontal elliptical dipole of a length equal to a half wavelength, and it is elevated from the ground by a distance which is about a quarter wavelength.

The explained design procedure (1)-(3) results in an antenna which covers the whole UWB range from $3.1 \mathrm{GHz}$ to 10.6 GHz. If it is required to reject a certain subband within 
that range, a slotline resonator can be incorporated in the feeder of the antenna in the manner shown in Figure 1(a). Configuration of the resonator is shown in Figure 1(b). Length of the slotline resonator is assumed to be equal to a quarter of the effective wavelength calculated at the center of the rejected band.

To understand effect of the slotline used in this paper, a transmission line model of the coplanar waveguide and the slotline resonator is shown in Figure 1(c). The resonator is equivalent to a short-circuited end series stub. At the center of the rejected band, the stub's length is equal to a quarter wavelength which means that the stub appears effectively as an open circuit at its point of connection with the main transmission line, which is the coplanar waveguide for the design presented in this paper. This comes from the fact that the input impedance of a quarter-wavelength stub is equal to $Z_{o}^{2} / Z_{t}$ [13], where $Z_{o}$ is the characteristic impedance of the stub and $Z_{t}$ is the terminal impedance of the stub, which is equal to zero in the stub considered in this paper. At the frequencies, which are far away from the resonance frequency of the stub, the resonator has negligible effect on the performance and the feeder and hence, the antenna performs as if there is no resonator.

It is to be noted that in order to use the space available for the stub efficiently and to make the antenna compact in size, the stub is folded in the manner shown in Figure 1(b).

\section{RESULTS}

The proposed UWB antenna was designed using GML1032 substrate with a dielectric constant equal to 3.2, tangent loss $\tan \delta=0.004$, and thickness of $1.52 \mathrm{~mm}$. Values of the design parameters for the antenna and the incorporated resonator were first calculated using the proposed design procedure and then optimized using the software Ansoft HFSSv10. The optimized values are: $w=l=27 \mathrm{~mm}, s=2.9 \mathrm{~mm}$, $w_{c}=0.25 \mathrm{~mm}, g=0.5 \mathrm{~mm}, l_{r}=11 \mathrm{~mm}, w_{s}=0.3 \mathrm{~mm}$, and $s_{r}=0.1 \mathrm{~mm}$.

Two samples of the proposed antenna were manufactured, one without and the other with the slotline resonator. Characteristics of the developed antennas were tested via simulations using the software HFSSv10 and via measurements using a vector network analyser in an anechoic chamber. Figure 2 shows variation of the SWR with frequency for the developed antennas. The simulated and measured characteristics of the antenna without a resonator reveal UWB behavior with bandwidth from $3 \mathrm{GHz}$ to more than $11 \mathrm{GHz}$ assuming $\mathrm{SWR}=2$ (or $10 \mathrm{~dB}$ return loss) as a reference. It is also clear from Figure 2 that, for the antennas with a resonator, the undesired subband was tuned out, whereas the wideband behavior of the antenna was maintained. The simulated and measured results are in good agreement.

It is worthwhile to mention that the slot width of the resonator $s_{r}$ can be used to adjust width of the rejected subband. A parametric analysis using the software HFSS shows that increasing $s_{r}$ increases width of the rejected band and its level of rejection, that is, a higher VSWR at the rejected band with a larger slot width. Moreover, the

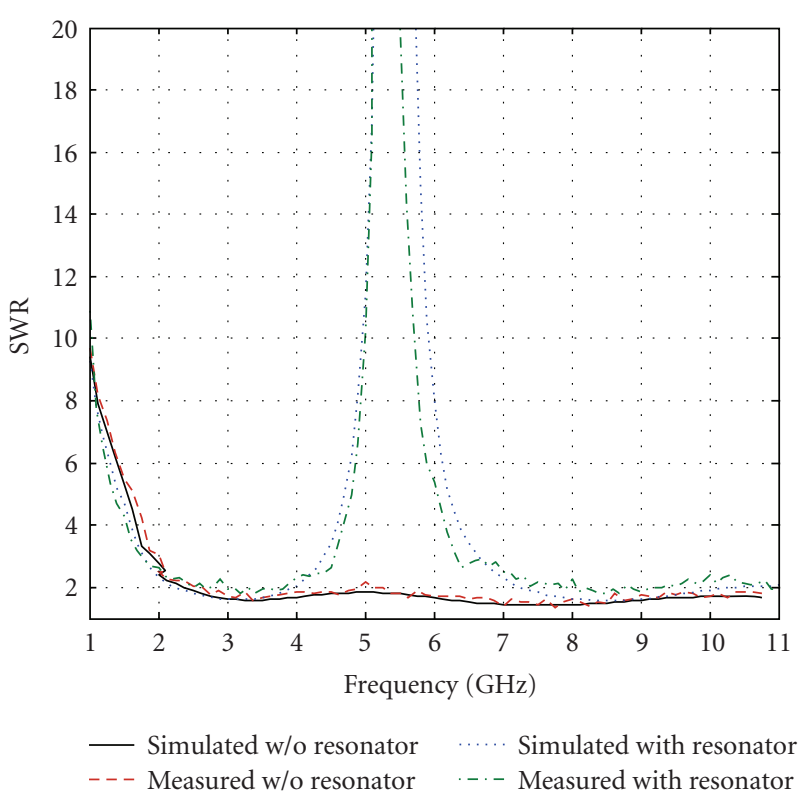

FIGURE 2: Variation of the SWR with frequency.

parametric analysis shows that width of the part of the feeder bounded by the resonator, that is, $w_{s}$ in Figure 1(b), has a significant effect on the level of rejection. Optimization techniques can be utilized to achieve a certain compromise between the required width of the rejected band and the level of rejection.

From the UWB applications point of view, the antenna is usually required to have an omnidirectional radiation. Concerning the designed antenna, this requirement is fulfilled over the whole bandwidth as shown in Figure 3 for the antenna without a resonator. The radiation patterns of the antenna with a resonator are similar to the results shown in Figure 3 and therefore, they are not shown here.

Figure 4 shows variation of the measured gain of the developed antennas across the ultra-wideband. It is clear from the results in Figure 4 that the antennas have a low gain, which agrees with the expected behavior of the omnidirectional antennas. The gain of the antenna without a resonator increases with frequency from around $0 \mathrm{dBi}$ at $3.1 \mathrm{GHz}$ to $3 \mathrm{dBi}$ at the range $9-11 \mathrm{GHz}$. Concerning gain of the developed antenna with the slotline resonator, the measured results, presented in Figure 4, show that the general behavior of the gain is similar to that of the antenna without a resonator except for the rejected subband. Across the band $4.9 \mathrm{GHz}$ to $5.9 \mathrm{GHz}$, the gain can be as low as $-8 \mathrm{dBi}$, when a resonator is used, compared with $2.2 \mathrm{dBi}$ for the antenna without a resonator. This proves the high capability of the proposed resonator to reject the undesired subband.

The last investigation concerns variation of the radiation efficiency for the designed antennas with and without the resonator. Results of the calculations using the software HFSS indicated that the proposed antennas feature a good efficiency, being greater than $92 \%$ across the desired band. 


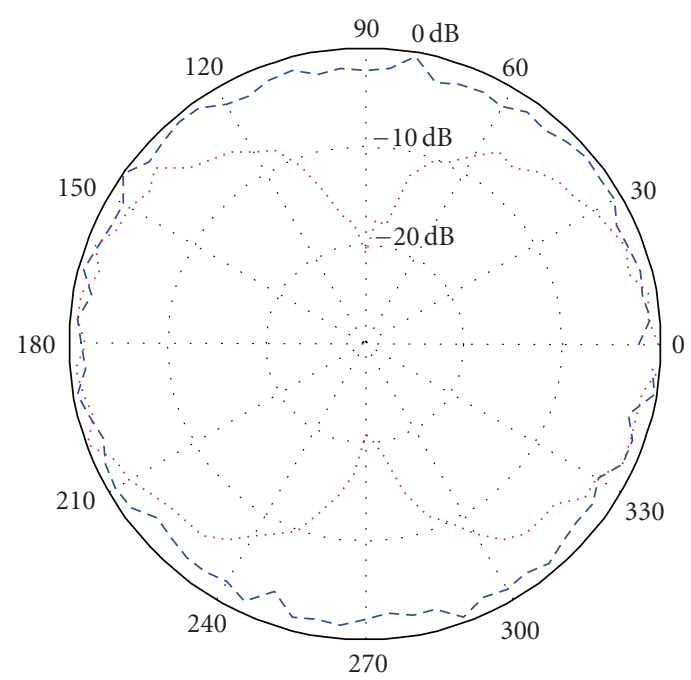

- - - xz plane

..... $y z$ plane

(a)

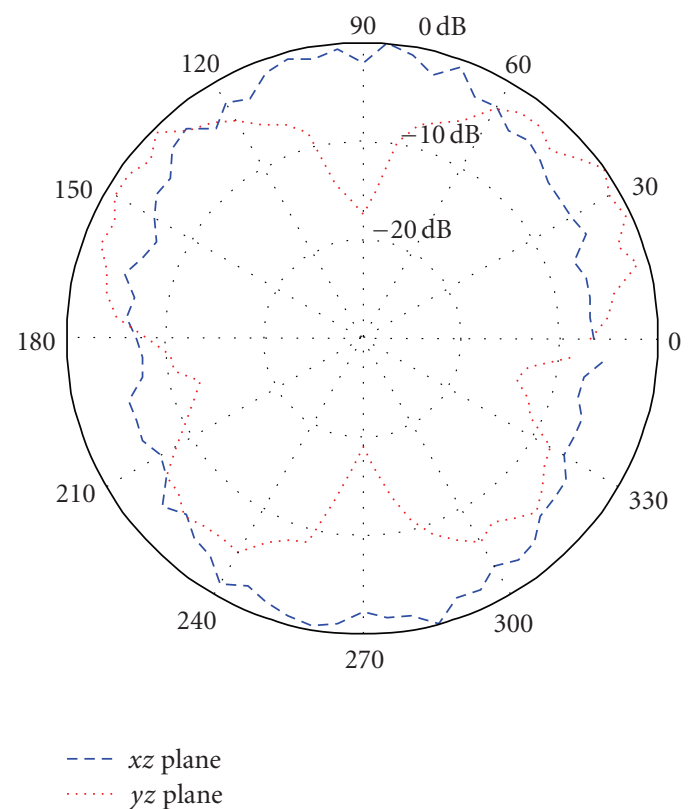

(b)

FIGURE 3: The measured radiation pattern of the antenna at (a) $3 \mathrm{GHz}$ and (b) $6 \mathrm{GHz}$.

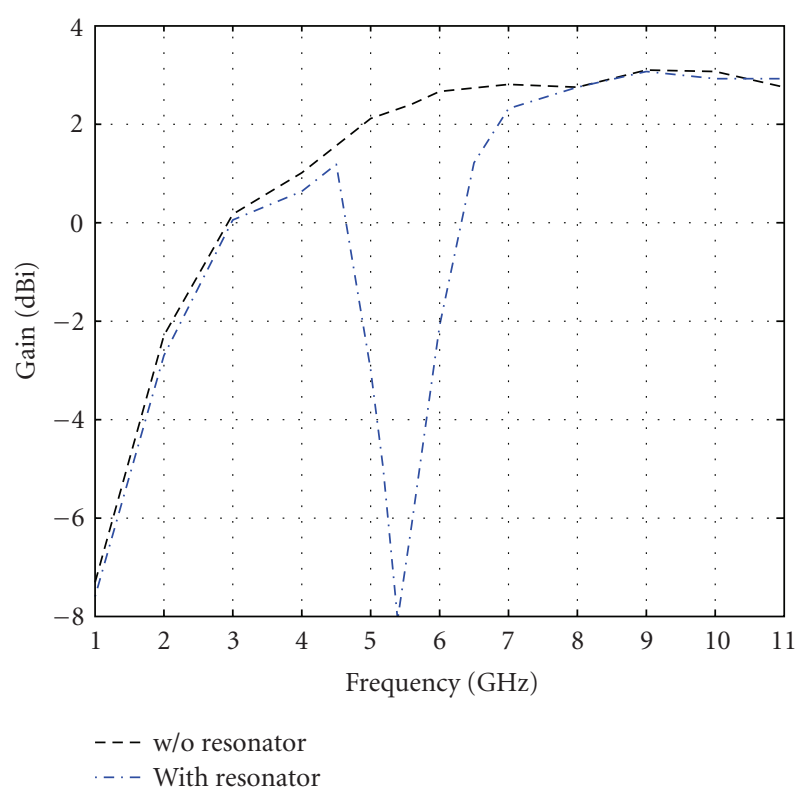

FIGURE 4: Variation of the measured gain with frequency for the manufactured antennas.

\section{CONCLUSION}

This paper has presented a complete design method for a compact ultra-wideband antenna with band-notched characteristics. The subband rejection is accomplished by incorporating a slotline resonator in the coplanar waveguide feeder of the antenna. To validate the proposed method, two samples of the proposed antenna were designed and manufactured: one does not contain a resonator, whereas the other contains a slotline resonator. Results of the simulation and measurement have shown that the designed antennas have an ultra-wideband performance with bandwidth from $3 \mathrm{GHz}$ to more than $11 \mathrm{GHz}$. The results have also shown effectiveness of the resonator in rejecting any undesired band, such as the $4.9-5.9 \mathrm{GHz}$ band, where the gain of the antenna with the resonator was reduced by more than $10 \mathrm{~dB}$ at the rejected band.

\section{ACKNOWLEDGMENT}

The author acknowledges the financial support of the University of Queensland via the postdoctoral research fellowship.

\section{REFERENCES}

[1] "Report and Order in the Commission's Rules Regarding Ultra-Wideband Transmission Systems," Released by Federal Communications Commission, April 2002.

[2] A. J. Kerkhoff and H. Ling, "Design of a planar monopole antenna for use with ultra-wideband (UWB) having a bandnotched characteristic," in Proceedings of IEEE Antennas and Propagation Society International Symposium (AP-S '03), vol. 1, pp. 830-833, Columbus, Ohio, USA, June 2003.

[3] A. J. Kerkhoff and H. Ling, "Design of a band-notched planar monopole antenna using genetic algorithm optimization," IEEE Transactions on Antennas and Propagation, vol. 55, no. 3, pp. 604-610, 2007.

[4] H. G. Schantz, G. Wolenec, and E. M. Myszka III, "Frequency notched UWB antennas," in Proceedings of IEEE Conference on 
Ultra Wideband Systems and Technologies (UWBST '03), pp. 214-218, Reston, Va, USA, November 2003.

[5] A. M. Abbosh, M. E. Bialkowski, J. Mazierska, and M. V. Jacob, "A planar UWB antenna with signal rejection capability in the 4-6 GHz band," IEEE Microwave and Wireless Components Letters, vol. 16, no. 5, pp. 278-280, 2006.

[6] Y.-J. Cho, K.-H. Kim, D. H. Choi, S. S. Lee, and S.-O. Park, "A miniature UWB planar monopole antenna with $5 \mathrm{GHz}$ bandrejection filter and the time-domain characteristics," IEEE Transactions on Antennas and Propagation, vol. 54, no. 5, pp. 1453-1460, 2006.

[7] C.-Y. Huang and W.-C. Hsia, "Planar ultra-wideband antenna with a frequency notch characteristic," Microwave and Optical Technology Letters, vol. 49, no. 2, pp. 316-320, 2007.

[8] S.-Y. Suh, W. L. Stutzman, W. A. Davis, A. E. Waltho, K. W. Skeba, and J. L. Schiffer, "A UWB antenna with a stopband notch in the $5 \mathrm{GHz}$ WLAN band," in Proceedings of IEEE/ACES International Conference on Wireless Communications and Applied Computational Electromagnetics, pp. 203 207, Honolulu, Hawaii, USA, April 2005.

[9] Y. Gao, B.-L. Ooi, and A. P. Popov, "Band-notched ultrawideband ring-monopole antenna," Microwave and Optical Technology Letters, vol. 48, no. 1, pp. 125-126, 2006.

[10] K.-H. Kim, Y.-J. Cho, S.-H. Hwang, and S.-O. Park, "Bandnotched UWB planar monopole antenna with two parasitic patches," Electronics Letters, vol. 41, no. 14, pp. 783-785, 2005.

[11] W. J. Lui, C. H. Cheng, and H. B. Zhu, "Frequency notched printed slot antenna with parasitic open-circuit stub," Electronics Letters, vol. 41, no. 20, pp. 1094-1095, 2005.

[12] K. Gupta, R. Garg, I. Bahl, and P. Bhartis, Microstrip Lines and Slotlines, Artech House, Boston, Mass, USA, 2nd edition, 1996.

[13] D. Pozar, Microwave Engineering, John Wiley \& Sons, New York, NY, USA, 3rd edition, 2005. 

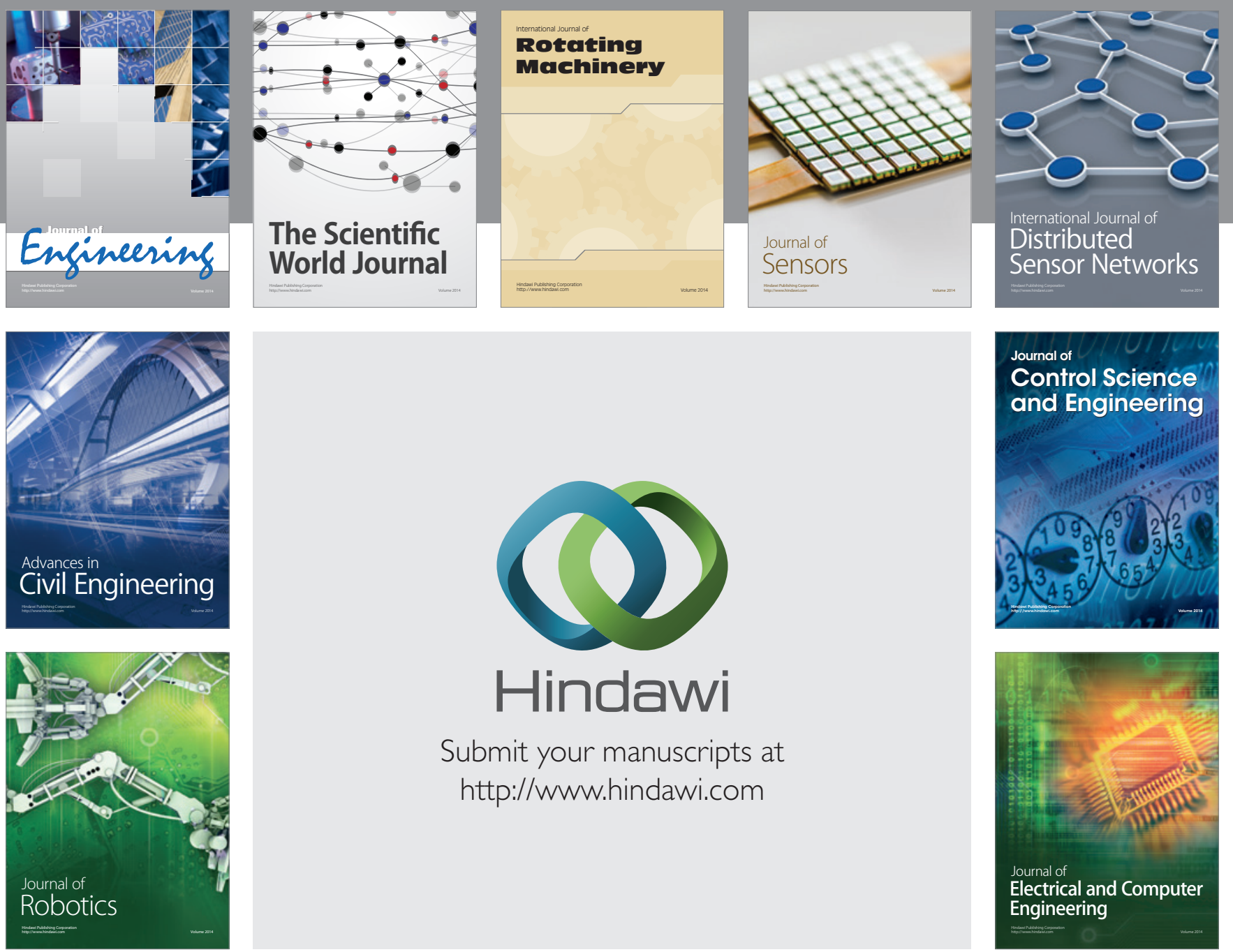

Submit your manuscripts at

http://www.hindawi.com
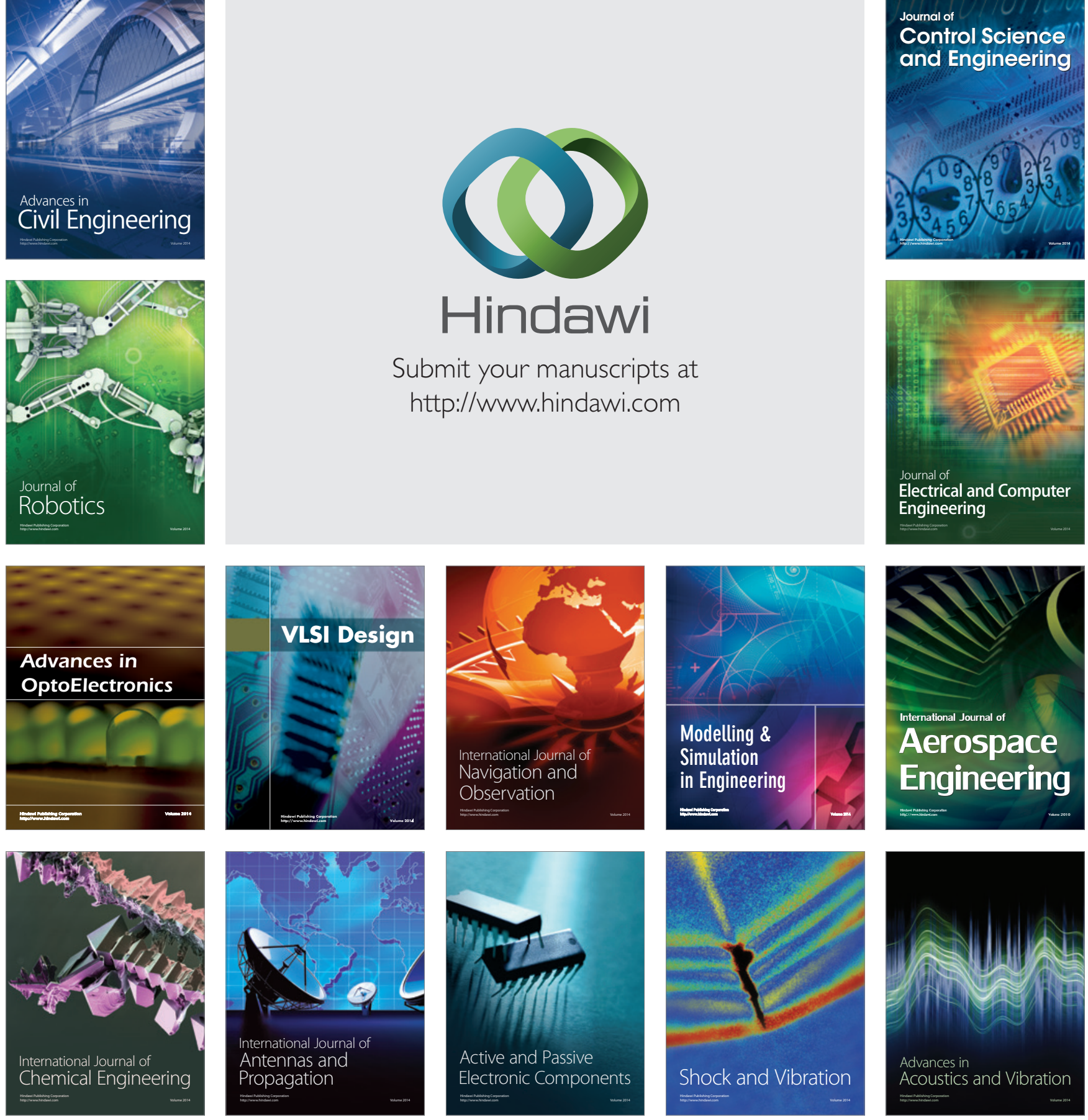